\title{
Host genetic variants potentially associated with SARS-CoV-2 A multi-population analysis
}

Maria K. Smatti ${ }^{1,2}$, Yasser A. Al-Sarraj², Omar Albagha ${ }^{2,3^{\star}}$, Hadi M. Yassine ${ }^{1,2^{\star}}$

${ }^{1}$ Biomedical Research Center, ${ }^{2} \mathrm{Hamad}$ Bin Khalifa University, ${ }^{3}$ University of Edinburgh

\section{INTRODUCTION}

Clinical outcomes of coronavirus disease 2019 (COVID-19), caused by the severe acute respiratory syndrome coronavirus 2 (SARS-CoV-2) showed enormous inter-individual and inter-population differences, possibly due to host genetics differences. Earlier studies identified single nucleotide polymorphisms (SNPs) associated with SARS-CoV-1 in Eastern Asian (EAS) populations. Importantly, these variants could have a potential effect on SARS-CoV-2 outcomes.
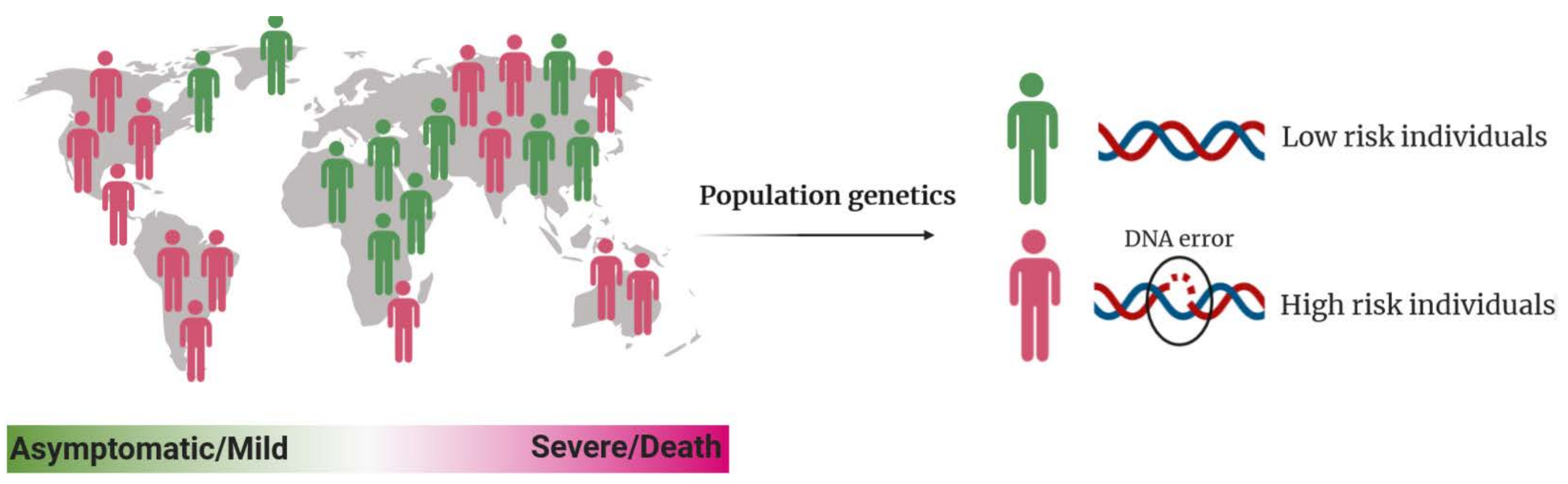

Study Aim: To explore the frequency of a set of genetic polymorphisms that could affect SARS-CoV-2 susceptibility or severity in the Qatari population, in comparison to other populations

\section{METHODS}

1 SNPs selection

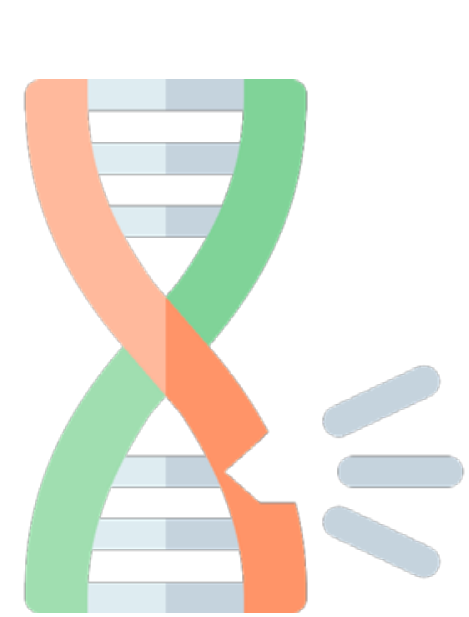

SNPs that were previously associated with SARS-CoV-1 were extracted from Genome wide association studies (GWAS)

Study population and Genomics data

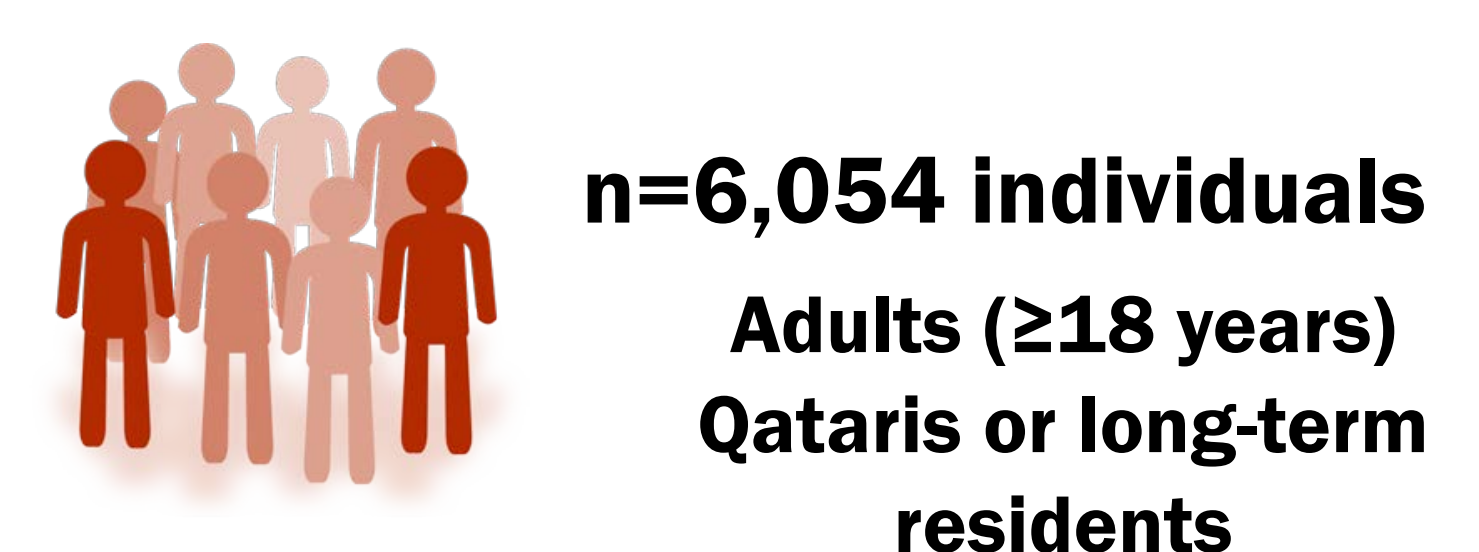
residents

Whole-genome sequences individuals who had previously participated in Qatar Genome Project (QGP) were obtained
(3) Analysis
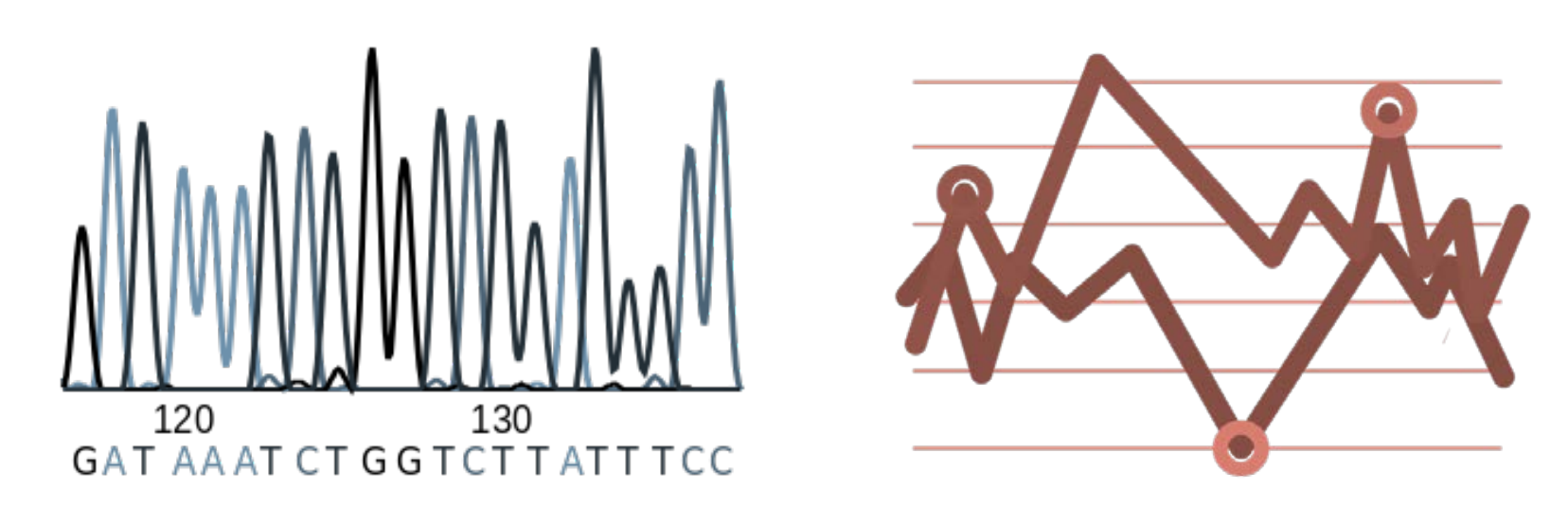

Sequences were used to calculate the allelic frequencies (AF) and compare it to the global AF from the $1000 \mathrm{Genome}$ project $(n=2504)$

\section{RESULTS}

1 Allelic frequencies of SARS-CoV-1-associated genetic variants among different populations

\begin{tabular}{ccc}
\hline Gene & SNP ID & AF 1000Genome \\
\hline$I L 4$ & rs2070874 & 0.4012 \\
CD209 & rs4804803 & 0.2334 \\
\hline AHSG & rs2248690 & 0.2398 \\
\hline CCL5 & rs2280788 & 0.0245 \\
\hline CCL2 & rs1024611 & 0.3636 \\
\hline MBL & rs1800450 & 0.122 \\
\hline TMPRSS2 & rs2070788 & 0.3968 \\
\hline TMPRSS2 & rs383510 & 0.3964 \\
\hline Furin & rs4932178 & 0.2644 \\
\hline ICAM3 & rs2304237 & 0.1795 \\
\hline IFN- $\gamma$ & rs2430561 & 0.7198 \\
\hline
\end{tabular}

$\triangle$ Higher susceptibility to SARS-CoV $\nabla$ Lower susceptibility to SARS-CoV
2 Fold differences in the risk allelic frequencies of SARS-CoV-1related SNPs among Qatari population in comparison to 1000Genome and Eastern Asian populations.

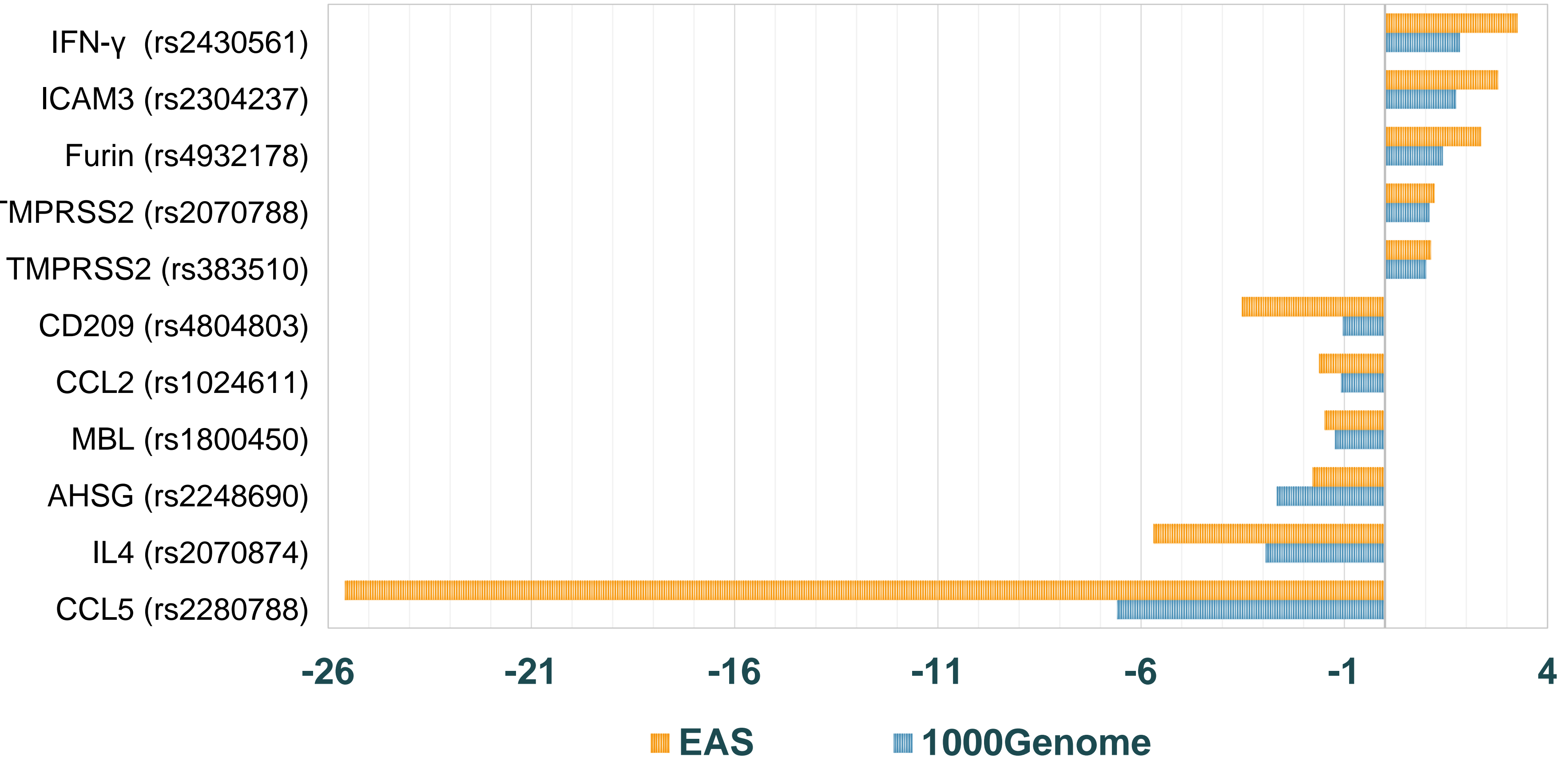

